\title{
RADIOGRAPHIC ASSESSMENT OF MANDIBULAR SYMMETRY FOLLOWING RECONSTRUCTION OF SEGMENTAL MANDIBULAR DEFECTS
}

\author{
Adel Hamdy Abou-ElFetouh* and Yasmine Ahmed Nassar*
}

\begin{abstract}
Restoration of symmetric facial contours after resection of mandibular tumors is one of the requisites of a successful reconstructive surgery. For unilateral mandibular defects and using the "mirroring" feature available in many surgical simulation software, it is possible to restore the undistorted mandibular contours and bend the reconstruction plate prior to surgery.
\end{abstract}

The aim of this study: is to assess whether pre-bent mandibular reconstruction plates using computer-assisted techniques for post tumor-ablation defects are good enough at restoring mandibular symmetry radiographically.

Patients and Methods: eight patients with segmental mandibular post-tumor resection defects, to whom a computer assisted planning and pre-bending of the reconstruction plates were performed; were included in this study. For each patient, on the 3D reformatted image, a midsagittal plane was constructed and the perpendicular distances were measured from the midsagittal plane to the titanium reconstruction plate and to the contralateral intact side on the axial cuts. The mean differences calculated and statistically analyzed.

Results: The mean of the differences between the intact and reconstructed sides of the mandible showed normal data distribution. Overall mean among all patients was $0.7225 \mathrm{~mm} \pm 0.24467$. Onesample $t$ test against a hypothetical mean difference of $0 \mathrm{~mm}$ revealed an extremely statistically significant difference between the intact and reconstructed sides with a $p$-value $<0.0001$.

Conclusion: Computer-assisted precontoured mandibular reconstruction plates can be considered to offer a very good degree of symmetry during mandibular reconstruction procedures.

\section{INTRODUCTION}

Mandibular reconstruction after resection of benign tumors remains to be a highly demanding procedure with an aim of restoring aesthetics, function and the quality of life. Restoration of symmetric facial contours after resection of mandibular tumors is one of the requisites of a successful reconstructive surgery ${ }^{1}$.

* Lecturer, Oral and Maxillofacial Surgery Department, Faculty of Oral and Dental Medicine - Cairo University 
A tumor-affected mandible is usually deformed to a significant degree that precludes the possibility of intraoperatively bending a reconstruction plate onto the mandible prior to accomplishing the resection. Attempts to contour a reconstruction plate after the resection is completed might risk improper seating of the condyle in the glenoid fossa with resultant malocclusion and temporomandibular disorders. To overcome this problem, some authors recommended the use of various types of condylarpositioning devices (CPDs) to maintain the condyle in place while a reconstruction plate is being adapted and fixed ${ }^{2,3}$. However, CPDs add to the overall cost and length of the surgery.

It was not until the introduction of computerassisted techniques to the field of oral and maxillofacial surgery that a true solution for this problem has been proposed. For unilateral mandibular defects and using the "mirroring" feature available in many surgical simulation software, it is possible to restore the undistorted mandibular contours followed by fabrication of a physical model by additive manufacturing techniques for pre-bending a mandibular reconstruction plate pl, $^{4,6}$.

Through this study, we aim to assess whether pre-bent mandibular reconstruction plates using computer-assisted techniques for post tumorablation defects are good enough at restoring mandibular symmetry radiographically.

\section{PATIENTS AND METHODS}

Patients were selected for inclusion in this study during the period from January 2013 to August 2017 from the department of Oral and Maxillofacial Surgery, Cairo University. The inclusion criteria were: segmental unilateral mandibular defects; either right or left side; and restoration of mandibular continuity using a titanium reconstruction plate. Whereas exclusion criteria were: free-hand bent and adapted reconstruction plates, patients with resultant soft tissue defects and patients with missing postoperative computed tomography (CT) or whose CTs were not available in a Digital Imaging and Communication in Medicine (DICOM) format.

Eight patients could be identified who fulfilled the applied criteria. For these patients, DICOM data of their postoperative CTs were imported into a surgical simulation software*. The CT data were then realigned based on the Frankfurt plane.

On the 3D reformatted image, a midsagittal plane was constructed ( $f i g$ 1). Subsequently, perpendicular distances were measured from the midsagittal plane to the titanium reconstruction plate and to the contralateral intact side on the axial cuts ( $f i g$ ). The difference between the two recorded measurements was calculated. Three measurementswere recorded along the anteroposterior (AP) plane and the differences calculated.

A mean of the three calculated measurements differences was obtained for each patient. The means were checked for normality using the Shapiro Wilk test $^{7}$. Assuming perfect symmetry between both sides of a mandible, a hypothetical mean difference of zero millimeters was assumed between the intact and reconstructed sides against which the obtained results were analyzed using a one-sample $t$ test.

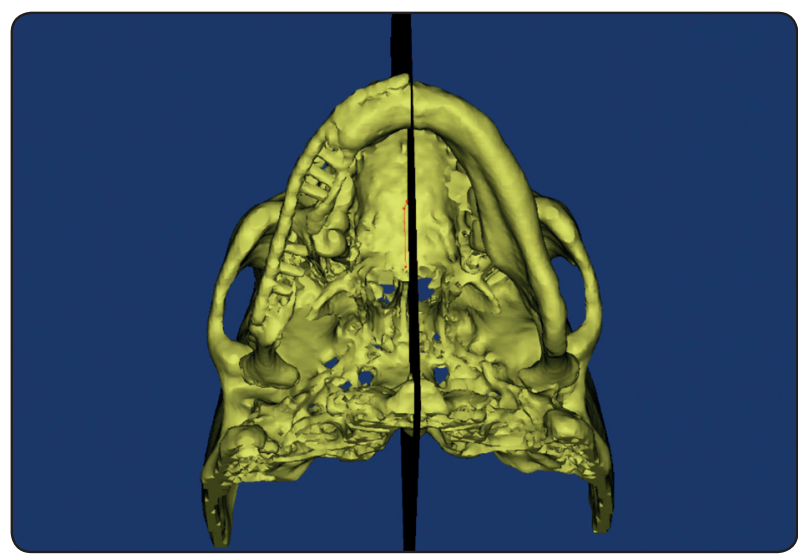

Fig. (1) Mid-sagittal plane construction on the reformatted 3D image of the skull.

* Mimics 15.0, Materialise Software, Leuven, Belgium 


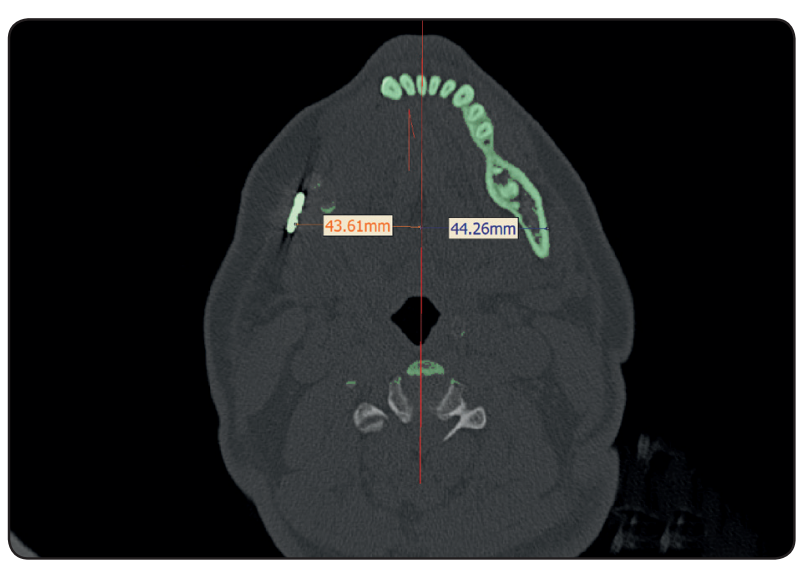

Fig. (2) Measurements of the distance from the constructed mid-sagittal mandible to the intact and reconstructed sides of the mandible.

\section{RESULTS}

Eight patients were included in this study, descriptive data of the included sample can be found in table 1. The mean of the differences between the intact and reconstructed sides of the mandible

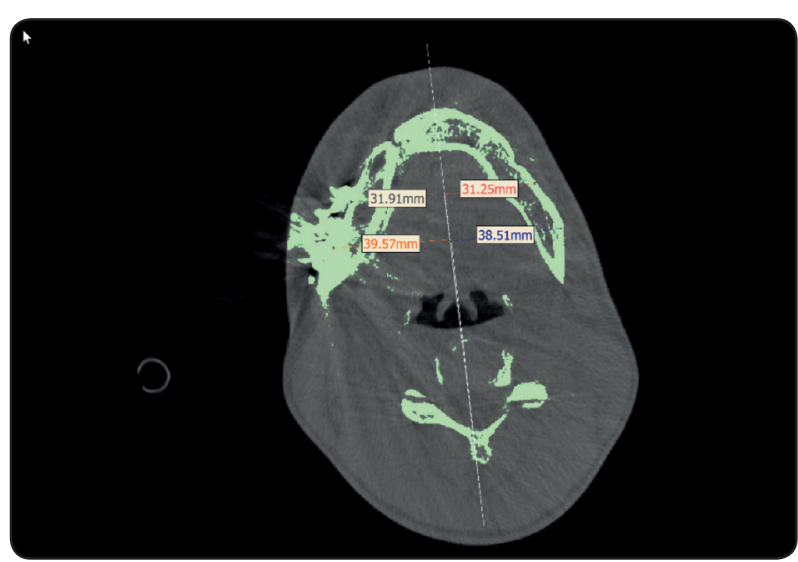

Fig. (3) Another case: measurements from the constructed midsagittal plane to the unaffected and reconstructed sides of the mandible

showed normal data distribution. Overall mean among all patients was $0.7225 \mathrm{~mm} \pm 0.24467$. Onesample $t$ test against a hypothetical mean difference of $0 \mathrm{~mm}$ revealed an extremely statistically significant difference between the intact and reconstructed sides with a p-value $<0.0001$

TABLE (1) Descriptive data of the patient sample included in the study.

\begin{tabular}{|c|c|c|c|c|c|c|c|c|}
\hline & Patient 1 & Patient 2 & Patient 3 & Patient 4 & Patient 5 & Patient 6 & Patient 7 & Patient 8 \\
\hline Difference 1 & 0.58 & 0.99 & 0.34 & 0.31 & 0.72 & 0.6 & 0.48 & 1.12 \\
\hline Difference 2 & 1.1 & 0.65 & 0.45 & 0.34 & 0.94 & 0.65 & 0.66 & 1.05 \\
\hline Difference 3 & 1.05 & 0.83 & 0.65 & 0.4 & 1.2 & 0.55 & 0.74 & 0.94 \\
\hline Mean Difference & 0.91 & 0.823333 & 0.48 & 0.35 & 0.953333 & 0.6 & 0.626667 & 1.036667 \\
\hline $\begin{array}{c}\text { Overall mean } \\
\text { Difference }\end{array}$ & & & & & & & & \\
\hline $\begin{array}{c}\text { Standard } \\
\text { Deviation }\end{array}$ & & & & & & & & \\
\hline
\end{tabular}




\section{DISCUSSION}

Even though a true facial symmetry between the right and left sides do not really exist, however, mirroring of the intact onto the affected side is a powerful planning tool that has been reported in many applications in the cranio-maxillofacial field ${ }^{8,9}$. It can be argued that the degree of dissymmetry that normally exists between the right and left sides of the face ${ }^{10}$ is clinically unrecognizable and thus "mirroring" can be comfortably used for virtually planning reconstruction of unilateral defects.

This can perfectly explain that although a mean difference of $0.7225 \mathrm{~mm}$ has been calculated among our sample, all these patients were clinically judged as to have very good to excellent facial contours as proven in their records. The highly statistically significant difference obtained by the one-sample $t$ test can be attributed to the hypothetical; yet not true; assumption of a perfect symmetry between both sides of the mandible. This statistical finding could have changed remarkably if the hypothetical difference between both sides of the mandible would have been assigned a value other than zero millimeters.

Pre-contoured reconstruction plates have the added advantages of shortening the surgical time, and if added to other computer-generated CPDs to allow for a more reproducible occlusion and less $\mathrm{TMD}^{11}$.

A very important limitation of computerassisted techniques becomes evident in patients with defects that cross the mandibular mid-line. For these patients, there is no intact side that one could virtually use to mirror-image onto the other side. In such patients, the surgeon might revert back to traditional free-hand techniques or hopefully as the technology evolves more we might eventually end up with a library of facial bones classified according to sex, age and race from which the surgeon can choose one with a "best fit".
Despite being accurately pre-contoured based on a computer-generated model, yet accurate placement and fixation of the reconstruction plate to the mandible as in the virtual plan cannot be guaranteed. One of the advocated tools to help guide the surgeon's way through to accurate installation of the plate as per the virtual plan is dynamic real-time control using navigation techniques ${ }^{12}$. However, navigation is a very expensive tool to implement and is not widely available specially in developing countries. Whether navigation would offer any more accuracy of clinical value over other "static" computer-assisted techniques is a point that needs further investigation.

\section{CONCLUSIONS}

Computer-assisted precontoured mandibular reconstruction plates can be considered to offer a very good degree of symmetry during mandibular reconstruction procedures.

\section{REFERENCES}

1. Eufinger H, Wehmöller M, Machtens E. Individual prostheses and resection templates for mandibular resection and reconstruction. Br J Oral Maxillofac Surg. Dec; 35(6):413-8.1997

2. Yagami K, Nagumo M. A transoral approach for threedimensional repositioning of the proximal segment after mandibular sagittal split ramus osteotomy. J OralMaxillofac Surg. Oct;54(10):1256-8.1996

3. Harada K, Ono J, Okada Y, Nagura H, Enomoto S. Postoperative stability after sagittal split ramus osteotomy with condylar-positioning appliance and screw fixation: asymmetric versus symmetric cases. Oral Surg Oral Med Oral Pathol Oral RadiolEndod. May;83(5):532-6. 1997

4. Hallermann W, Olsen S, Bardyn T, Taghizadeh F, Banic A, Iizuka T. A new method for computer-aided operation planning for extensive mandibular reconstruction. PlastReconstr Surg. Jun; 117(7):2431-7. 2006

5. Hannen EJ. Recreating the original contour in tumor deformed mandibles for plate adapting. Int J Oral Maxillofac Surg. Feb;35(2):183-5. 2006 
6. Lee JW, Fang JJ, Chang LR, Yu CK. Mandibular defect reconstruction with thehelp of mirror imaging coupled with laser stereolithographic modeling technique.J Formos Med Assoc. Mar;106(3):244-50. 2007

7. http://sdittami.altervista.org/shapirotest/ShapiroTest.html

8. Francaviglia N, Maugeri R, OdiernaContino A, Meli F, Fiorenza V, Costantino G, Giammalva RG, Iacopino DG. Skull Bone Defects reconstruction with Custom-Made Titanium Graft shaped with Electron Beam Melting Technology: Preliminary Experience in a Series of Ten Patients. ActaNeurochir Suppl. 124:137-141. doi: 10.1007/978-3319-39546-3_21. 2017

9. Scolozzi P. Applications of 3D orbital computer-assisted surgery (CAS). J Stomatol Oral Maxillofac Surg. Sep; 118(4):217-223. doi: 10.1016/j.jormas.2017.05.007. 2017
10. Belcastro A, Willing R, Jenkyn T, Johnson M, Galil K, Yazdani A. A Three-dimensional Analysis of Zygomatic Symmetry in Normal, Uninjured Faces. J Craniofac Surg. Mar;27(2):504-8. doi: 10.1097/SCS.0000000000002210. 2016

11. Abou-ElFetouh A, Barakat A, Abdel-Ghany K. Computerguided rapid-prototyped templates for segmental mandibular osteotomies: a preliminary report. Int J Med Robot. Jun;7(2):187-92. 2011

12. Li P, Xuan M, Liao C, Tang W, Wang XY, Tian W, Long J. Application of Intraoperative Navigation for the Reconstruction of Mandibular Defects With Microvascular Fibular Flaps-Preliminary Clinical Experiences. J Craniofac Surg. May;27(3):751-5. doi: 10.1097/ SCS.0000000000002430. 2016 